\title{
Women and Cardiac Disease: A Special Issue
}

\author{
Roxana Mehran ${ }^{(1)}$ and Birgit Vogel ${ }^{(1)}$ \\ Icahn School of Medicine at Mount Sinai, New York - USA
}

Cardiovascular disease is the leading cause of death in women worldwide. Despite improvements in awareness on heart disease in women through various campaigns and initiatives, cardiovascular risk in women still tends to be underestimated by health care professionals and women themselves. With respect to the latter, a study from the United States has even shown a decline in awareness regarding the fact that heart disease is the leading cause of death among women over recent years. ${ }^{1}$ Especially young women are affected by low awareness about cardiovascular risk as confirmed by the data from the Variation in Recovery: Role of Gender on Outcomes of Young AMI Patients (VIRGO) study, which also found that women are less likely than men to have a discussion about their cardiovascular risk and potential risk modification with their health care provider. ${ }^{2}$ Health care providers' preparedness to assess women's cardiovascular risk appears to be suboptimal, and according to survey data the majority of primary care physicians and cardiologists do not comprehensively implement cardiovascular disease prevention guidelines in women. ${ }^{3}$ Accurate and timely diagnosis of various cardiovascular diseases, especially ischemic heart disease, are less frequent in women compared with men. In addition, women with established cardiovascular

\section{Keywords}

International Journal of Cardiovascular Sciences; Heart Diseases; Women.

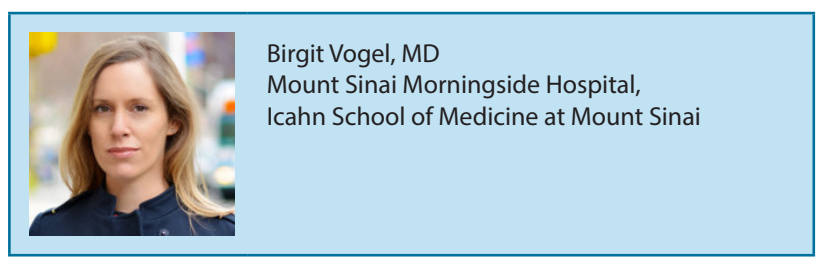

disease have repeatedly been documented to be less likely to receive guideline-recommended therapies. ${ }^{4,5}$ Sex-related differences in disease presentation and comorbidities may contribute to these disparities, and uncertainties persist with respect to diagnosis and treatment of certain disease states that disproportionally affect women (e.g. ischemia with non-obstructive coronary arteries [INOCA], myocardial infarction in the absence of obstructive coronary artery disease [MINOCA], spontaneous coronary artery dissection [SCAD], and takotsubo syndrome).

One of the major issues is the lack of robust study data on cardiovascular disease in women. Women remain underrepresented in the majority of cardiovascular clinical trials, and sex-specific study data reporting is often absent, which results in uncertainty about the safety and efficacy of many cardiovascular therapies. ${ }^{6}$ In addition, it leads to knowledge gaps in the sex-specific pathophysiological mechanisms and the natural history of cardiovascular disease in women.

Cardiovascular risk factors may affect women differently than men, and there is growing evidence about risk factors specific to women, including premature menopause and pregnancy-related disorders. ${ }^{7}$ Some studies also suggest that the association between cardiovascular risk and socioeconomic deprivation is more pronounced in women compared with men. Further investigation is urgently needed to evaluate the sex-specific impact of well-established risk factors, sex-specific conditions, and

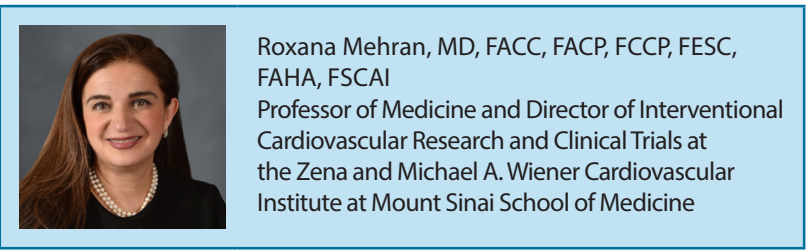


the direct and indirect effects of underrecognized factors, such psychosocial factors, on women's cardiovascular health. It should be one of the major research targets to better understand how to incorporate these factors in risk stratification tools and how to improve risk prediction, especially in young women.

Further research is also needed to investigate the role of sex hormones on cardiovascular risk in women. It is well documented that, in comparison with men, women generally present later in life with coronary artery disease and that cardiovascular risk increases significantly after menopause. ${ }^{8}$ While data suggest that cardiovascular risk and the development of cardiovascular disease are impacted by endogenous and exogenous reproductive hormone differences, it is still not fully elucidated how sex-hormones affect the cardiovascular system in women. Despite the lower cardiovascular risk of young (premenopausal) women compared with their male counterparts, alarming trends have recently been documented, with a rise in myocardial infarction in young women ${ }^{9}$ and an overall increase in cardiovascular disease mortality in women from certain countries. ${ }^{10}$ It must be a priority to investigate the underlying reasons and find strategies to oppose these worrisome developments.

\section{References}

1. Cushman M, Shay CM, Howard VJ, Jiménez MC, Lewey J, McSweeney JC, et al. Ten-Year Differences in Women's Awareness Related to Coronary Heart Disease: Results of the 2019 American Heart Association National Survey: A Special Report From the American Heart Association. Circulation. 2021;143(7):239-48. doi: 10.1161/CIR.0000000000000907.

2. Leifheit-Limson EC, D'Onofrio G, Daneshvar M, Geda M, Bueno H, Spertus JA, et al. Sex Differences in Cardiac Risk Factors, Perceived Risk, and Health Care Provider Discussion of Risk and Risk Modification Among Young Patients with Acute Myocardial Infarction: The VIRGO Study. J Am Coll Cardiol. 2015;66(18):1949-57. doi: 10.1016/j.jacc.2015.08.859.

3. Merz CNB, Andersen H, Sprague E, Burns A, Keida M, Walsh MN, et al. Knowledge, Attitudes, and Beliefs Regarding Cardiovascular Disease in Women: The Women's Heart Alliance. J Am Coll Cardiol. 2017;70(2):123-32. doi: 10.1016/j.jacc.2017.05.024.

4. Redfors B, Angerås O, Råmunddal T, Petursson P, Haraldsson I, Dworeck C, et al. Trends in Gender Differences in Cardiac Care and Outcome After Acute Myocardial Infarction in Western Sweden: A Report from the Swedish Web System for Enhancement of Evidence-Based Care in Heart Disease Evaluated According to Recommended Therapies (SWEDEHEART). J Am Heart Assoc. 2015;4(7):e001995. doi: 10.1161/ JAHA.115.001995.

5. Udell JA, Fonarow GC, Maddox TM, Cannon CP, Peacock WF, Laskey WK, et al. Sustained Sex-Based Treatment Differences in Acute Coronary
This special issue of the International Journal of Cardiovascular Sciences on cardiac disease in women features important research in women and heart disease; the original research articles include studies providing valuable insights on cardiovascular risk factors, risk classification, and risk profiles of women from certain age groups and geographical areas. Furthermore, this issue comprises data on women traversing menopause, which marks a critical time of cardiovascular risk acceleration in a woman's life, and it addresses cardiovascular risk in the growing population of breast cancer survivors. A comprehensive literature review on sex and gender differences in ischemic heart disease as well as interesting case reports are among the other high-yield articles in this issue. Providing a platform for research on cardiovascular disease in women in this way is a crucial step to inform the medical and scientific communities, to raise awareness, and to motivate further studies on this important topic. A robust and comprehensive evidence base on cardiovascular disease in women is the cornerstone of providing optimal care to women and reducing the global burden of cardiovascular disease. This special issue on cardiac disease in women is an important effort in addressing the gender gap and developing sex-specific strategies for improved prevention, diagnosis, and treatment of cardiovascular disease worldwide.

Syndrome Care: Insights from the American Heart Association get with The Guidelines Coronary Artery Disease Registry. Clin Cardiol. 2018;41(6):758-68. doi: 10.1002/clc.22938.

6. Jin X, Chandramouli C, Allocco B, Gong E, Lam CSP, Yan LL. Women's Participation in Cardiovascular Clinical Trials From 2010 to 2017. Circulation. 2020;141(7):540-8. doi: 10.1161/ CIRCULATIONAHA.119.043594.

7. Agarwala A, Michos ED, Samad Z, Ballantyne CM, Virani SS. The Use of Sex-Specific Factors in the Assessment of Women's Cardiovascular Risk. Circulation. 2020;141(7):592-9. doi: 10.1161/ CIRCULATIONAHA.119.043429.

8. Yusuf S, Hawken S, Ounpuu S, Dans T, Avezum A, Lanas F, et al. Effect of Potentially Modifiable Risk Factors Associated with Myocardial Infarction in 52 Countries (the INTERHEART study): Case-Control Study. Lancet. 2004;364(9438):937-52. doi: 10.1016/S0140-6736(04)17018-9.

9. Arora S, Stouffer GA, Kucharska-Newton AM, Qamar A, Vaduganathan M, Pandey A, et al. Twenty Year Trends and Sex Differences in Young Adults Hospitalized with Acute Myocardial Infarction. Circulation. 2019;139(8):1047-56. doi: 10.1161/CIRCULATIONAHA.118.037137.

10. Lopez AD, Adair T. Is the Long-Term Decline in Cardiovascular-Disease Mortality in High-Income Countries Over? Evidence from National Vital Statistics. Int J Epidemiol. 2019;48(6):1815-23. doi: 10.1093/ije/dyz143. 